\title{
Reverse Poincaré inequalities, Isoperimetry, and Riesz transforms in Carnot groups
}

Fabrice Baudoin, Michel Bonnefont

October 15, 2018

\begin{abstract}
We prove an optimal reverse Poincaré inequality for the heat semigroup generated by the sub-Laplacian on a Carnot group of any step. As an application we give new proofs of the isoperimetric inequality and of the boundedness of the Riesz transform in Carnot groups.
\end{abstract}

\section{Contents}

1 Introduction

2 The optimal reverse Poincaré inequality for the heat semigroup in Carnot groups

2.1 Preliminaries on Carnot groups . . . . . . . . . . . . . . . 3

2.2 The optimal reverse Poincaré inequality . . . . . . . . . . . . . . 7

2.3 The case of H-type groups . . . . . . . . . . . . . . . . 9

3 Isoperimetric inequality 11

4 Boundedness of the Riesz transform 13

\section{Introduction}

In this paper, we are interested in reverse Poincaré inequalities for Markov semigroups. If $P_{t}$ is a Markov semigroup generated by a diffusion operator $L$, such an inequality writes in the form of

$$
\Gamma\left(P_{t} f\right) \leq C(t)\left(P_{t} f^{2}-\left(P_{t} f\right)^{2}\right)
$$

where $C(t)$ is a positive constant and $\Gamma$ the carré $d u$ champ operator:

$$
\Gamma(f)=\frac{1}{2}\left(L f^{2}-2 f L f\right) .
$$


This inequality is a regularization estimate in the sense that it allows to control derivatives of $P_{t} f$ in terms of the sup-norm of $f$ only. If $L$ is the Laplace operator on $\mathbb{R}^{n}$, it is easy to check that the inequality (1.1) holds with $C(t)=\frac{1}{2 t}$, and this is the best possible constant.

The reverse Poincaré inequality is closely related to intrinsic curvature properties of the generator $L$. Actually, it turns out that the inequality

$$
\Gamma\left(P_{t} f\right) \leq \frac{K}{e^{2 K t}-1}\left(P_{t} f^{2}-\left(P_{t} f\right)^{2}\right)
$$

is equivalent to the Bakry-Émery criterion

$$
\Gamma_{2}(f) \geq K \Gamma(f)
$$

where

$$
\Gamma_{2}(f)=\frac{1}{2}(L \Gamma(f)-2 \Gamma(f, L f))
$$

is the usual Bakry's $\Gamma_{2}$ operator. For further details on this, we refer to the book by BakryGentil-Ledoux [3]. As an example, on a complete Riemannian manifold, the inequality

$$
\left\|\nabla P_{t} f\right\|^{2} \leq \frac{K}{e^{2 K t}-1}\left(P_{t} f^{2}-\left(P_{t} f\right)^{2}\right)
$$

is satisfied for the semigroup generated by the Laplace-Beltrami operator, if and only if the Ricci curvature of the manifold is bounded from below by $K$.

As is it now understood, the Bakry-Émery criterion requires some form of ellipticity for the generator and typically fails to hold for strictly hypoelliptic operators [2, 4]. However. if $L$ is a hypoelliptic diffusion operator, the semigroup $P_{t}$ is smoothing in the sense that it transforms bounded Borel functions into smooth functions. For this reason, it is natural to expect, that under suitable conditions an inequality like (1.1) should hold. In [2], it was proved that if $L$ is the sub-Laplacian in the Heisenberg group, the following reverse Poincaré inequality holds

$$
\Gamma\left(P_{t} f\right) \leq \frac{1}{t}\left(P_{t} f^{2}-\left(P_{t} f\right)^{2}\right) .
$$

Moreover, the constant $\frac{1}{t}$ is optimal. In the same reference, it was conjectured that a similar inequality should hold on any Carnot group. In the present paper, we prove that this is indeed the case and moreover compute the optimal constant of the inequality.

The paper is organized as follows. In Section 2, we first recall some basic results about Carnot groups and their heat semigroups and then proceed to work out the optimal reverse Poincaré inequality. In Section 3, we show how the reverse Poincaré inequality implies the isoperimetric inequality. Finally in Section 4, we give a second application of the reverse Poincaré inquality, by showing that the Riesz transform on Carnot groups is bounded. 


\section{The optimal reverse Poincaré inequality for the heat semi- group in Carnot groups}

\subsection{Preliminaries on Carnot groups}

A Carnot group of step (or depth) $N$ is a simply connected Lie group $\mathbb{G}$ whose Lie algebra can be written

$$
\mathfrak{g}=\mathcal{V}_{1} \oplus \ldots \oplus \mathcal{V}_{N}
$$

where

$$
\left[\mathcal{V}_{i}, \mathcal{V}_{j}\right]=\mathcal{V}_{i+j}
$$

and

$$
\mathcal{V}_{s}=0, \text { for } s>N \text {. }
$$

From the above properties, it is of course seen that Carnot groups are nilpotent. The number

$$
Q=\sum_{i=1}^{N} i \operatorname{dim} \mathcal{V}_{i}
$$

is called the homogeneous dimension of $\mathbb{G}$.

\section{Example 2.1}

1. (Commutative case) The group $\left(\mathbb{R}^{d},+\right)$ is the only commutative Carnot group.

2. (Heisenberg groups) Consider the set $\mathbb{H}_{n}=\mathbb{R}^{2 n} \times \mathbb{R}$ endowed with the group law

$$
(x, \alpha) \star(y, \beta)=\left(x+y, \alpha+\beta+\frac{1}{2} \omega(x, y)\right),
$$

where $\omega$ is the standard symplectic form on $\mathbb{R}^{2 n}$, that is

$$
\omega(x, y)=x^{t}\left(\begin{array}{cc}
0 & -\mathbf{I}_{n} \\
\mathbf{I}_{n} & 0
\end{array}\right) y .
$$

On $\mathfrak{h}_{n}$ the Lie bracket is given by

$$
[(x, \alpha),(y, \beta)]=(0, \omega(x, y)),
$$

and it is easily seen that

$$
\mathfrak{h}_{n}=\mathcal{V}_{1} \oplus \mathcal{V}_{2},
$$

where $\mathcal{V}_{1}=\mathbb{R}^{2 n} \times\{0\}$ and $\mathcal{V}_{2}=\{0\} \times \mathbb{R}$. Therefore $\mathbb{H}_{n}$ is a Carnot group of depth 2. 
3. (Engel group) The Engel group is the 4-dimensional Lie group of matrices

$$
\mathbb{E}=\left\{\left(\begin{array}{cccc}
1 & x & \frac{x^{2}}{2} & z \\
0 & 1 & x & w \\
0 & 0 & 1 & y \\
0 & 0 & 0 & 1
\end{array}\right), x, y, w, z \in \mathbb{R}\right\}
$$

Its Lie algebra $\mathfrak{e}$ is generated by the matrices

$$
\begin{gathered}
X=\left(\begin{array}{llll}
0 & 1 & 0 & 0 \\
0 & 0 & 1 & 0 \\
0 & 0 & 0 & 0 \\
0 & 0 & 0 & 0
\end{array}\right), Y=\left(\begin{array}{llll}
0 & 0 & 0 & 0 \\
0 & 0 & 0 & 0 \\
0 & 0 & 0 & 1 \\
0 & 0 & 0 & 0
\end{array}\right) \\
W=\left(\begin{array}{llll}
0 & 0 & 0 & 0 \\
0 & 0 & 0 & 1 \\
0 & 0 & 0 & 0 \\
0 & 0 & 0 & 0
\end{array}\right), Z=\left(\begin{array}{llll}
0 & 0 & 0 & 1 \\
0 & 0 & 0 & 0 \\
0 & 0 & 0 & 0 \\
0 & 0 & 0 & 0
\end{array}\right),
\end{gathered}
$$

for which we have the following structure relations,

$$
[X, Y]=W, \quad[X, W]=Z
$$

and all other brackets are zero. The Engel group is easily seen to be a Carnot group of step 3.

On $\mathfrak{g}$ we can consider the family of linear operators $\delta_{t}: \mathfrak{g} \rightarrow \mathfrak{g}, t \geq 0$ which act by scalar multiplication $t^{i}$ on $\mathcal{V}_{i}$. These operators are Lie algebra automorphisms due to the grading. The maps $\delta_{t}$ induce Lie group automorphisms $\Delta_{t}: \mathbb{G} \rightarrow \mathbb{G}$ which are called the canonical dilations of $\mathbb{G}$. It is easily seen that there exists on $\mathbb{G}$ a complete and smooth vector field $D$ such that

$$
\Delta_{t}=e^{(\ln t) D}
$$

This vector field $D$ is called the dilation vector field on $\mathbb{G}$. If $X$ is a left (or right) invariant smooth horizontal vector field on $\mathbb{G}$, that is $X(0) \in \mathcal{V}_{1}$, we have for every $f \in C^{\infty}(\mathbb{G})$, and $t \geq 0$,

$$
X\left(f \circ \Delta_{t}\right)=t X f
$$

Let us now pick a basis $V_{1}, \ldots, V_{d}$ of the vector space $\mathcal{V}_{1}$. The vectors $V_{i}$ 's can be seen as left invariant vector fields on $\mathbb{G}$. In the sequel, these vector fields shall still be denoted by $V_{1}, \ldots, V_{d}$ and the corresponding right invariant vector fields shall be denoted by $\hat{V}_{1}, \ldots, \hat{V}_{d}$. The left invariant sub-Laplacian on $\mathbb{G}$ is the operator:

$$
L=\sum_{i=1}^{d} V_{i}^{2} .
$$


It is essentially self-adjoint on the space of smooth and compactly supported function $f: \mathbb{G} \rightarrow \mathbb{R}$ with the respect to the Haar measure $\mu$ of $\mathbb{G}$. The heat semigroup $\left(P_{t}\right)_{t \geq 0}$ on $\mathbb{G}$, defined through the spectral theorem, is then seen to be a Markov semigroup (see [14]). This heat semigroup admits a positive fundamental solution named the heat kernel and denoted by $p_{t}\left(g, g^{\prime}\right)$. We often simply denote $p_{t}(g)=p_{t}(0, g)$ for the heat kernel issued from the identity. By left invariance, it is enough to know this heat kernel issued from the identity to recover all the heat kernels. Since Carnot groups enjoy the volume doubling property and carry the Poincaré inequality on balls, we deduce that $p_{t}$ satisfies the double-sided Gaussian bounds (see Theorem 2.9 in [14]):

$$
\frac{C^{-1}}{t^{Q / 2}} \exp \left(-\frac{C_{1} d(0, g)^{2}}{t}\right) \leq p_{t}(g) \leq \frac{C}{t^{Q / 2}} \exp \left(-C_{2} \frac{d(0, g)^{2}}{t}\right),
$$

for some constants $C, C_{1}, C_{2}>0$. Here $d(0, g)$ is Carnot-Carathéodory distance from 0 to $g$ in $\mathbb{G}$.

As usual, if $f: \mathbb{G} \rightarrow \mathbb{R}$ is a smooth function, we denote,

$$
\Gamma(f, f)=\sum_{i=1}^{d}\left(V_{i} f\right)^{2}
$$

This is the carré du champ operator of $L$. Sometimes, as a shortened notation we will denote $\Gamma(f)$ for $\Gamma(f, f)$. The following gradient bound can be found in [14, Theorem 2.7,

$$
\sqrt{\Gamma\left(p_{t}\right)}(g) \leq \frac{C}{t^{\frac{Q+1}{2}}} \exp \left(-C_{3} \frac{d(0, g)^{2}}{t}\right) .
$$

We also introduce the right-invariant sub-Laplacian:

$$
\hat{L}=\sum_{i=1}^{d} \hat{V}_{i}^{2}
$$

and we denote by $\hat{P}_{t}$ the associated heat semigroup and by $\hat{p}_{t}$ the associated heat kernel. We also denote

$$
\hat{\Gamma}(f, f)=\sum_{i=1}^{d}\left(\hat{V}_{i} f\right)^{2}
$$

First, we begin with two useful lemmas, whose proofs are classical and let to the reader. The main argument is that for $f \in L^{2}(\mathbb{G}, \mu), P_{t} f$ is the unique solution in $L^{2}(\mathbb{G}, \mu)$ of the parabolic Cauchy problem:

$$
\left\{\begin{array}{l}
\frac{\partial \phi}{\partial t}=L \phi \\
\phi(0, x)=f(x)
\end{array}\right.
$$

The same characterization holds for $\hat{P}_{t}$. Our first lemma relies the two semigroups $P_{t}$ and $\hat{P}_{t}$. 
Lemma 2.2 Let $f \in L^{2}(\mathbb{G}, \mu)$. Then for $g \in \mathbb{G}$, one has:

$$
\hat{P}_{t}(f)(g)=P_{t}\left(f \circ A d\left(g^{-1}\right)\right)(g)
$$

where $A d\left(g^{-1}\right)$ is the function defined by $A d\left(g^{-1}\right)(h)=g^{-1} h g$. As a consequence, one has:

$$
\hat{P}_{t}(f)(0)=P_{t}(f)(0),
$$

and for every $g \in \mathbb{G}$

$$
\hat{p}_{t}(g)=p_{t}(g) .
$$

The second lemma illustrates the scaling property of the semigroup $P_{t}$ with respect to the dilations. The second identity of the lemma can be obtained by differentiating the first one at $c=1$.

Lemma 2.3 Let $f \in L^{2}(\mathbb{G}, \mu)$. For every $t, c \geq 0$,

$$
P_{t}\left(f \circ \Delta_{\sqrt{c}}\right)=\left(P_{c t} f\right) \circ \Delta_{\sqrt{c}} .
$$

Moreover, if $f \in C_{0}^{\infty}(\mathbb{G})$, then for every $t \geq 0$,

$$
P_{t} D f=D P_{t} f+t P_{t} L f .
$$

We conclude this preliminary section with an integrability lemma which shall be useful in the sequel.

Lemma 2.4 Let $f: \mathbb{G} \rightarrow \mathbb{R}$ be a smooth function with polynomial growth, that is,

$$
|f(g)| \leq C(1+d(0, g))^{N}, \quad g \in \mathbb{G},
$$

for some $C>0, N \geq 0$, then for $t>0$,

$$
\int_{\mathbb{G}} f p_{t} \Gamma\left(\ln p_{t}, \ln p_{t}\right) d \mu<+\infty .
$$

Proof. As the dilation vector field $D$ vanishes at 0 , for all $t>0$ and for any smooth and compactly supported $h$,

$$
P_{t}((t L-D) h)(0)=0
$$

that is

$$
\int_{\mathbb{G}}(t L-D) h p_{t} d \mu=0
$$

This implies

$$
\left(t L+D+\frac{Q}{2}\right) p_{t}=0
$$

because the the adjoint $D^{*}$ of $D$ is $-D-\frac{Q}{2}$. 
Let now $h \in C_{0}^{\infty}(\mathbb{G})$. We have

$$
\begin{aligned}
\int_{\mathbb{G}} h f p_{t} \Gamma\left(\ln p_{t}, \ln p_{t}\right) d \mu & =\int_{\mathbb{G}} h f \Gamma\left(\ln p_{t}, p_{t}\right) d \mu \\
& =\int_{\mathbb{G}} \Gamma\left(h f \ln p_{t}, p_{t}\right) d \mu-\int_{\mathbb{G}} \ln p_{t} \Gamma\left(h f, p_{t}\right) d \mu \\
& =-\int_{\mathbb{G}} h f \ln p_{t} L p_{t} d \mu-\int_{\mathbb{G}} \ln p_{t} \Gamma\left(h f, p_{t}\right) d \mu \\
& =-\frac{1}{t} \int_{\mathbb{G}} D\left(h f \ln p_{t}\right) p_{t} d \mu-\int_{\mathbb{G}} \ln p_{t} \Gamma\left(h f, p_{t}\right) d \mu
\end{aligned}
$$

In exponential coordinates, the vector fields $D$ and $V_{i}$ 's have polynomial coefficients. We can thus easily construct an increasing sequence $h_{n} \in C_{0}^{\infty}(\mathbb{G})$ such that $0 \leq h_{n} \leq 1, h_{n} \rightarrow 1$ and $\left|D h_{n}\right|(g) \leq \frac{1}{n} P(g), \sqrt{\Gamma}\left(h_{n}\right)(g) \leq \frac{1}{n} P(g)$, where $P$ is a function with polynomial growth on $\mathbb{G}$. We now use the previous equalities with $h_{n}$ in place of $h$. We have

$$
\int_{\mathbb{G}} D\left(h_{n} f \ln p_{t}\right) p_{t} d \mu=\int_{\mathbb{G}} h_{n} D\left(f \ln p_{t}\right) p_{t} d \mu+\int_{\mathbb{G}} D\left(h_{n}\right) f p_{t} \ln p_{t} d \mu .
$$

Thus, from the bounds (2.2) and (2.3), by dominated convergence, we obtain

$$
\int_{\mathbb{G}} D\left(h_{n} f \ln p_{t}\right) p_{t} d \mu \rightarrow \int_{\mathbb{G}} D\left(f \ln p_{t}\right) p_{t} d \mu .
$$

Similarly, we have

$$
\int_{\mathbb{G}} \ln p_{t} \Gamma\left(h_{n} f, p_{t}\right) d \mu \rightarrow \int_{\mathbb{G}} \ln p_{t} \Gamma\left(f, p_{t}\right) d \mu .
$$

\subsection{The optimal reverse Poincaré inequality}

We now turn to the inequality which is of interest for us. The following reverse Poincaré inequality for the heat semigroup is optimal.

Proposition 2.5 Let $f: \mathbb{G} \rightarrow \mathbb{R}$ be a smooth and compactly supported function. For $g \in \mathbb{G}$,

$$
\Gamma\left(P_{t} f, P_{t} f\right)(g) \leq \frac{\Lambda}{t}\left(P_{t} f^{2}(g)-\left(P_{t} f\right)^{2}(g)\right), \quad t>0 .
$$

where the constant $\Lambda$ is the largest eigenvalue of the symmetric matrix

$$
M=\left(\int_{\mathbb{G}} \hat{V}_{i}\left(\ln p_{1}\right) \hat{V}_{j}\left(\ln p_{1}\right) p_{1} d \mu\right)_{1 \leq i, j \leq d} .
$$

Moreover, the constant $\Lambda$ is sharp. 
Proof. By left invariance and scaling, it is enough to check it at the identity and $t=1$. Let $f: \mathbb{G} \rightarrow \mathbb{R}$ be a smooth and compactly supported function, then

$$
\Gamma\left(P_{1} f, P_{1} f\right)(0)=\sup _{\sum_{i=1}^{d} a_{i}^{2}=1}\left(\sum_{i=1}^{d} a_{i} V_{i} P_{1}(f)(0)\right)^{2} .
$$

Now, let $a_{i} \in \mathbb{R}$ be such that $\sum_{i=1}^{d} a_{i}^{2}=1$, then

$$
\begin{aligned}
\sum_{i=1}^{d} a_{i} V_{i} P_{1}(f)(0) & =P_{1}\left(\sum_{i=1}^{d} a_{i} \hat{V}_{i} f\right)(0) \\
& =-\int_{\mathbb{G}} \sum_{i=1}^{d} a_{i} \hat{V}_{i}\left(p_{1}\right) f d \mu \\
& =-\int_{\mathbb{G}} \sum_{i=1}^{d} a_{i} \hat{V}_{i}\left(\ln p_{1}\right) f p_{1} d \mu
\end{aligned}
$$

Therefore, by Cauchy-Schwarz inequality against the measure $p_{1} d \mu$, we have

$$
\left(\sum_{i=1}^{d} a_{i} V_{i} P_{1}(f)(0)\right)^{2} \leq \int_{\mathbb{G}}\left(\sum_{i=1}^{d} a_{i} \hat{V}_{i}\left(\ln p_{1}\right)\right)^{2} p_{1} d \mu \int_{\mathbb{G}} f^{2} p_{1} d \mu .
$$

But one can write:

$$
\begin{aligned}
\int_{\mathbb{G}}\left(\sum_{i=1}^{d} a_{i} \hat{V}_{i}\left(\ln p_{1}\right)\right)^{2} p_{1} d \mu & =\sum_{i, j=1}^{d} a_{i} a_{j} \int_{\mathbb{G}} \hat{V}_{i}\left(\ln p_{1}\right) \hat{V}_{j}\left(\ln p_{1}\right) p_{1} d \mu \\
& =A^{t} M A
\end{aligned}
$$

with $M$ the matrix defined in the proposition and $A=\left(a_{1}, \ldots a_{d}\right)^{t}$. The result follows then easily

The following proposition gives a lower and upper bound for the optimal constant $\Lambda$.

Proposition 2.6 We have

$$
\frac{Q}{2 d} \leq \Lambda \leq \frac{Q}{2}
$$

Proof. Since the matrix $M$ is symmetric, the constant $\Lambda$ satisfies the following inequality:

$$
\frac{1}{d} \text { trace } M \leq \Lambda \leq \operatorname{trace} M
$$

and the trace of $M$ is given by

$$
\operatorname{trace} M=\int_{\mathbb{G}} \hat{\Gamma}\left(\ln p_{1}\right) p_{1} d \mu=\int_{\mathbb{G}} \Gamma\left(\ln p_{1}\right) p_{1} d \mu .
$$


Thus, we just need to prove that

$$
\int_{\mathbb{G}} \Gamma\left(\ln p_{t}\right) p_{t} d \mu=\frac{Q}{2 t} .
$$

To this end, recall that

$$
\left(t L+D+\frac{Q}{2}\right) p_{t}=0
$$

Multiply then by $\ln p_{t}$ and taking integral gives

$$
\int_{\mathbb{G}} \ln p_{t}\left(t L+D+\frac{Q}{2}\right) p_{t} d \mu=0 .
$$

But by using an integration by parts (which we can justify as in Lemma 2.4), we have

$$
t \int_{\mathbb{G}} \ln p_{t} L p_{t} d \mu=-t \int_{\mathbb{G}} \Gamma\left(\ln p_{t}, \ln p_{t}\right) p_{t} d \mu .
$$

Moreover, we have

$$
\int_{\mathbb{G}} \ln p_{t}\left(D+\frac{Q}{2}\right) p_{t} d \mu=-\int_{\mathbb{G}} p_{t} D \ln p_{t} d \mu=-\int_{\mathbb{G}} D p_{t} d \mu=\frac{Q}{2} \int_{\mathbb{G}} p_{t} d \mu=\frac{Q}{2}
$$

and therefore

$$
\int_{\mathbb{G}} \Gamma\left(\ln p_{t}, \ln p_{t}\right) p_{t} d \mu=\frac{Q}{2 t}
$$

\subsection{The case of H-type groups}

We now prove that the lower bound is achieved in a special class of Carnot groups, the so-called $H$-type groups. For us, a $H$-type group will be simply $\mathbb{R}^{2 n+m}=\mathbb{R}^{2 n} \times \mathbb{R}^{m}$ equipped with the product

$$
v * w=v+w+\frac{1}{2}[v, w]
$$

where $[\cdot, \cdot]$ is a bracket operation on $\mathbb{R}^{2 n+m}$ whose center is $0 \times \mathbb{R}^{m}$ and such the map $J_{z}: \mathbb{R}^{2 n} \rightarrow \mathbb{R}^{2 n}$ defined for $z \in \mathbb{R}^{m}$ by:

$$
<J_{z}(x), y>=<[x, y], z>\text { for all } x, y \in \mathbb{R}^{2 n}
$$

is orthogonal when $|z|=1$. In the above we have identified $x \in \mathbb{R}^{2 n}$ with $(x, 0) \in \mathbb{R}^{2 n} \times \mathbb{R}^{m}$ and $z \in \mathbb{R}^{m}$ with $(0, z) \in \mathbb{R}^{2 n} \times \mathbb{R}^{m}$ and $|\cdot|$ denotes the classical Euclidean norm.

The left-invariant vector fields which concide with $\frac{\partial}{\partial x_{i}}$ at the identity write:

$$
V_{i}=\frac{\partial}{\partial x_{i}}+\frac{1}{2} \sum_{j=1}^{m}<J_{u_{j}} x, e_{i}>\frac{\partial}{\partial z_{j}}
$$


whereas the corresponding right-invariant vector fields write:

$$
\hat{V}_{i}=\frac{\partial}{\partial x_{i}}-\frac{1}{2} \sum_{j=1}^{m}<J_{u_{j}} x, e_{i}>\frac{\partial}{\partial z_{j}}
$$

for $i=1, \ldots, 2 n$ and where $\left(e_{1}, \ldots e_{2 n}\right)$ is the canonical basis of $\mathbb{R}^{2 n}$ and $\left(u_{1}, \ldots u_{m}\right)$ the canonical basis of $\mathbb{R}^{m}$. It is easy to see that it is a Carnot group of step 2. The Haar measure is just the Lebesgue measure and the heat kernel associated to $L=\sum_{i=1}^{2 n} V_{i}^{2}$ issued from the identity is given by (see for instance [8]):

$$
p_{t}(x, z)=\frac{1}{(2 \pi)^{m}} \frac{1}{(4 \pi)^{n}} \int_{\mathbb{R}^{m}} e^{i<\lambda, z>} e^{-\frac{|\lambda||x|^{2}}{4} \operatorname{coth}|\lambda| t}\left(\frac{|\lambda|}{\sinh |\lambda| t}\right)^{n} d \lambda
$$

and therefore is only a function of $|x|$ and $|z|$.

In the case of $H$-type groups, we obtain the more precise statement.

Corollary 2.7 Assume $\mathbb{G}$ to be an $H$-type group, then

$$
\Lambda=\frac{Q}{2 d}
$$

Proof. Let $\mathbb{G}$ be a $H$-type group. By the previous proposition, the only thing that we need to do is to see that, in this case, the matrix $M$ writes $\lambda I d$ for some $\lambda \in \mathbb{R}$. This will come from the fact that the heat kernel is a radial function, this means that it only depends on $|x|$ and $|z|$. Recall also that a $H$-type group can be identified with $\mathbb{R}^{2 n+m}$ and that the vector fields $V_{i}, i=1, \ldots, 2 n$ read:

$$
\hat{V}_{i}=\frac{\partial}{\partial x_{i}}-\frac{1}{2} \sum_{j=1}^{m}<J_{u_{j}} x, e_{i}>\frac{\partial}{\partial z_{j}}
$$

where $J_{u_{j}}$ is an orthogonal map of $\mathbb{R}^{2 n}$. Therefore the coefficient $M_{i j}$ of the matrix $M$ can be written as:

$$
\int_{x \in \mathbb{R}^{2 n}} \int_{z \in \mathbb{R}^{m}} \hat{V}_{i} f\left(|x|^{2},|z|^{2}\right) \hat{V}_{j} f\left(|x|^{2},|z|^{2}\right) g\left(|x|^{2},|z|^{2}\right) d x d z
$$

for some functions $f$ and $g$. Now,

$$
V_{i} f\left(|x|^{2},|z|^{2}\right)=2 x_{i} \partial_{1} f-\sum_{l=1}^{m} z_{l}<J_{u_{l}} x, e_{i}>\partial_{2} f
$$

and the result is coming by expanding the product and noticing that:

$$
\int_{x \in \mathbb{R}^{2 n}} x_{i} x_{j} h_{1}(|x|,|z|) d x=\delta_{i j} \tilde{h}_{1}(|z|)
$$




$$
\begin{gathered}
\int_{z \in \mathbb{R}^{m}} z_{j} h_{2}(|x|,|z|) d z=0, \\
\int_{z \in \mathbb{R}^{m}} z_{l} z_{p} h_{3}(x)=0 \text { for } l \neq p, \\
\int_{x \in \mathbb{R}^{2 n}} z_{l}^{2}<J_{u_{l}} x, e_{i}><J_{u_{l}} x, e_{j}>h_{4}(|x|,|z|) d x=\delta_{i j} z_{l}^{2} \tilde{h}_{4}(|z|)
\end{gathered}
$$

since $J_{u_{l}} x$ is an orthogonal map of $\mathbb{R}^{2 n}$ for $l=1, \ldots, m$ and that $\int_{z \in \mathbb{R}^{m}} z_{l}^{2} \tilde{h}_{4}(|z|) d z$ does not depend on $l$.

Note that the estimates in [8] show that all the integrals appearing in the above argument are well defined.

\section{Isoperimetric inequality}

In this section, we show that the reverse Poincaré inequality we proved in the previous section can be used to prove the isoperimetric inequality in Carnot groups. To this end, we adapt some beautiful ideas of Varopoulos (see [13, pp.256-258) and Ledoux (see pp. 22 in [10], see also Theorem 8.4 in [11])

In order to state the inequality we need the notion of horizontal perimeter, which is defined from the horizontal variation of a function.

Following [6], given a function $f \in L_{l o c}^{1}(\mathbb{G})$ we define the horizontal total variation of $f$ by

$$
\operatorname{Var}_{\mathcal{H}}(f)=\sup _{\phi \in \mathcal{F}(\mathbb{G})} \int_{\mathbb{G}} f\left(\sum_{i=1}^{d} V_{i} \phi_{i}\right) d \mu .
$$

where

$$
\mathcal{F}(\mathbb{G})=\left\{\phi \in C_{0}^{1}(\mathbb{G}, \mathcal{H}) \mid\|\phi\|_{\infty} \leq 1\right\} .
$$

Here, for $\phi=\sum_{i=1}^{d} \phi_{i} V_{i}$, we have let $\|\phi\|_{\infty}=\sup _{\mathbb{G}} \sqrt{\sum_{i=1}^{d} \phi_{i}^{2}}$.

The space

$$
B V_{\mathcal{H}}(\mathbb{G})=\left\{f \in L^{1}(\mathbb{G}) \mid \operatorname{Var}_{\mathcal{H}}(f)<\infty\right\},
$$

endowed with the norm

$$
\|f\|_{B V_{\mathcal{H}}(\mathbb{G})}=\|f\|_{L^{1}(\mathbb{G})}+\operatorname{Var}_{\mathcal{H}}(f)
$$

is a Banach space. One can note that when $f \in W_{\mathcal{H}}^{1,1}(\mathbb{G})$, then $f \in B V_{\mathcal{H}}(\mathbb{G})$, and one has in fact

$$
\operatorname{Var}_{\mathcal{H}}(f)=\|\sqrt{\Gamma(f)}\|_{L^{1}(\mathbb{G})}
$$

Given a measurable set $E \subset \mathbb{G}$ we say that it has finite horizontal perimeter if $\mathbf{1}_{E} \in$ $B V_{\mathcal{H}}(\mathbb{G})$. In such case we define the horizontal perimeter of $E$ by

$$
P_{\mathcal{H}}(E)=\operatorname{Var}_{\mathcal{H}}\left(\mathbf{1}_{E}\right) .
$$


We say that a measurable set $E \subset \mathbb{G}$ is a Caccioppoli set if $P_{\mathcal{H}}(E)<\infty$.

We now prove the following result which is due to Varopoulos.

Theorem 3.1 (Isoperimetric inequality) There is a constant $C_{\text {iso }}>0$, such that for every bounded Caccioppoli set $E \subset \mathbb{G}$

$$
\mu(E)^{\frac{Q-1}{Q}} \leq C_{\text {iso }} P_{\mathcal{H}}(E)
$$

Proof. Let $f \in C_{0}^{\infty}(\mathbb{G})$. From Proposition 2.5, we have

$$
\Gamma\left(P_{t} f\right) \leq \frac{\Lambda}{t}\|f\|_{L^{\infty}(\mathbb{G})}^{2}, \quad t>0
$$

Thus,

$$
\left\|\sqrt{\Gamma\left(P_{t} f\right)}\right\|_{L^{\infty}(\mathbb{G})} \leq \sqrt{\frac{\Lambda}{t}}\|f\|_{L^{\infty}(\mathbb{G})} .
$$

Applying this inequality to $g \in C_{0}^{\infty}(\mathbb{G})$, with $g \geq 0$ and $\|g\|_{L^{\infty}(\mathbb{G})} \leq 1$, if $f \in C_{0}^{1}(\mathbb{G})$ we have

$$
\begin{aligned}
\int_{\mathbb{G}} g\left(f-P_{t} f\right) d \mu & =\int_{0}^{t} \int_{\mathbb{G}} g \frac{\partial P_{s} f}{\partial s} d \mu d s=\int_{0}^{t} \int_{\mathbb{G}} g L P_{s} f d \mu d s=-\int_{0}^{t} \int_{\mathbb{G}} \Gamma\left(P_{s} g, f\right) d \mu d s \\
& \leq \int_{0}^{t}\left\|\sqrt{\Gamma\left(P_{s} g\right)}\right\|_{L^{\infty}(\mathbb{G})} \int_{\mathbb{G}} \sqrt{\Gamma(f)} d \mu d s \leq 2 \sqrt{\Lambda t} \int_{\mathbb{G}} \sqrt{\Gamma(f)} d \mu .
\end{aligned}
$$

We thus obtain the following basic inequality: for $f \in C_{0}^{1}(\mathbb{G})$,

$$
\left\|P_{t} f-f\right\|_{L^{1}(\mathbb{G})} \leq 2 \sqrt{\Lambda t}\|\sqrt{\Gamma(f)}\|_{L^{1}(G)}, \quad t>0 .
$$

Suppose now that $E \subset \mathbb{G}$ is a bounded Caccioppoli set. Therefore $\mathbf{1}_{E} \in B V_{\mathcal{H}}(\mathbb{G})$. By Theorem 1.14 in $[9]$. there exists a sequence $\left\{f_{n}\right\}_{n \in \mathbb{N}}$ in $C_{0}^{\infty}(\mathbb{G})$ satisfying

(i) $\left\|f_{n}-1_{E}\right\|_{L^{1}(\mathbb{G})} \rightarrow 0$;

(ii) $\int_{\mathbb{G}} \sqrt{\Gamma\left(f_{n}\right)} d \mu \rightarrow \operatorname{Var}_{\mathcal{H}}(f)$.

Applying (3.4) to $f_{n}$ we obtain

$$
\left\|P_{t} f_{n}-f_{n}\right\|_{L^{1}(\mathbb{G})} \leq 2 \sqrt{\Lambda t}\left\|\sqrt{\Gamma\left(f_{n}\right)}\right\|_{L^{1}(\mathbb{G})}=2 \sqrt{\Lambda t} \operatorname{Var}_{\mathcal{H}}\left(f_{n}\right), \quad n \in \mathbb{N} .
$$

Letting $n \rightarrow \infty$ in this inequality, we conclude

$$
\left\|P_{t} \mathbf{1}_{E}-\mathbf{1}_{E}\right\|_{L^{1}(\mathbb{G})} \leq 2 \sqrt{\Lambda t} \operatorname{Var}_{\mathcal{H}}\left(\mathbf{1}_{E}\right)=2 \sqrt{\Lambda t} P_{\mathcal{H}}(E), \quad t>0 .
$$

Observe now that we have

$$
\left\|P_{t} \mathbf{1}_{E}-\mathbf{1}_{E}\right\|_{L^{1}(\mathbb{G})}=2\left(\mu(E)-\int_{E} P_{t} \mathbf{1}_{E} d \mu\right) .
$$


On the other hand, we have

$$
\int_{E} P_{t} \mathbf{1}_{E} d \mu=\int_{\mathbb{M}}\left(P_{t / 2} \mathbf{1}_{E}\right)^{2} d \mu
$$

We thus obtain

$$
\left\|P_{t} \mathbf{1}_{E}-\mathbf{1}_{E}\right\|_{L^{1}(\mathbb{G})}=2\left(\mu(E)-\int_{\mathbb{G}}\left(P_{t / 2} \mathbf{1}_{E}\right)^{2} d \mu\right) .
$$

We now note that

$$
\begin{aligned}
\int_{\mathbb{G}}\left(P_{t / 2} \mathbf{1}_{E}\right)^{2} d \mu & \leq\left(\int_{E}\left(\int_{\mathbb{G}} p_{t / 2}(x, y)^{2} d \mu(y)\right)^{\frac{1}{2}} d \mu(x)\right)^{2} \\
& =\left(\int_{E} p_{t}(x, x)^{\frac{1}{2}} d \mu(x)\right)^{2} \leq \frac{p_{1}(0)}{t^{Q / 2}} \mu(E)^{2} .
\end{aligned}
$$

Combining these equations yields

$$
\mu(E) \leq \sqrt{\Lambda t} P_{\mathcal{H}}(E)+\frac{p_{1}(0)}{t^{Q / 2}} \mu(E)^{2}, \quad t>0 .
$$

Minimizing in $t$, we conclude

$$
\mu(E)^{\frac{Q-1}{Q}} \leq C P_{\mathcal{H}}(E)
$$

with

$$
C=(1+Q)^{\frac{Q+1}{Q}} p_{1}(0)^{\frac{1}{Q}} \frac{\Lambda}{Q}
$$

\section{Boundedness of the Riesz transform}

Besides the isoperimetric inequality, the reverse Poincaré for the heat semigroup is also intimately related to the boundedness of the Riesz transform. Actually combining Proposition 2.5 with the results in [5] which built on [1] leads to the following result.

Proposition 4.1 Let $1<p<\infty$. There exist constants $A_{p}, B_{p}>0$ such that

$$
A_{p}\left\|(-L)^{1 / 2} f\right\|_{p} \leq\|\sqrt{\Gamma(f)}\|_{p} \leq B_{p}\left\|(-L)^{1 / 2} f\right\|_{p}, \quad f \in C_{0}^{\infty}(\mathbb{G}),
$$

Proof. The main ingredient is Theorem 1.3 in [1]. The theorem is stated for Riemannian manifolds, but it is checked in [5] that the arguments go through in the context of CarnotCarathéodory spaces that satisfy the doubling condition and the Poincaré inequality. The only thing to check is the bound

$$
\left\|\sqrt{\Gamma} e^{t L}\right\|_{\infty \rightarrow \infty} \leq \frac{C}{\sqrt{t}}
$$


where $e^{t L}$ is the heat semigroup generated by $L$. This bound is a consequence of our Proposition 2.5.

We note that the first proof of the boundedness of the Riesz transform in Carnot groups can be found in [12].

\section{References}

[1] P. Auscher, T. Coulhon, X.T. Duong, S. Hofmann, Riesz transform on manifolds and heat kernel regularity, Ann. Sci. École Norm. Sup. (4) 37 (2004), no. 6, 911-957.

[2] D. Bakry, F. Baudoin, M. Bonnefont, D. Chafai: On gradient bounds for the heat kernel on the Heisenberg group. J. Funct. Anal. 255 (2008), no. 8, 1905-1938.

[3] D. Bakry, I. Gentil \& M. Ledoux: Analysis and Geometry of Markov Diffusion operators, Grundlehren der Mathematischen Wissenschaften [Fundamental Principles of Mathematical Sciences], 348. Springer, Cham, 2014. xx+552 pp

[4] F. Baudoin, M. Bonnefont, Log-Sobolev inequalities for subelliptic operators satisfying a generalized curvature dimension inequality, Journal of Functional Analysis, Volume 262, Issue 6, 2646-2676, 2012

[5] F. Baudoin \& N. Garofalo: A note on boundedness of Riesz transform for some subelliptic operators, International Mathematics Research Notices, (2013), no 2, 398421 .

[6] L. Capogna, D. Danielli \& N. Garofalo, The geometric Sobolev embedding for vector fields and the isoperimetric inequality, Comm. Anal. and Geom., 2 (1994), 201-215.

[7] B. Driver \& T. Melcher: Hypoelliptic heat kernel inequalities on the Heisenberg group. J. Funct. Anal. 221 (2005), no. 2, 340-365.

[8] N. Eldredge, Precise estimates for the subelliptic heat kernel on H-type groups. Journal de Mathématiques Pures et Appliquées 92 (2009), pp. 52-85.

[9] N. Garofalo \& D.M. Nhieu, Lipschitz continuity, global smooth approximations and extension theorems for Sobolev functions in Carnot-Carathéodory spaces, J. Anal. Math., 74 (1998), 67-97.

[10] M. Ledoux, Inégalités isopérimétriques en analyse et probabilités Séminaire Bourbaki. Astérisque 216, 343-375 (1993).

[11] M. Ledoux, Isoperimetry and Gaussian analysis Ecole d'été de Probabilités de StFlour 1994. Lecture Notes in Math. 1648, 165-294. Springer (1996).

[12] Lohoué, N., Varopoulos, N.: Remarques sur les transformées de Riesz sur les groupes nilpotents. C.R.A.S. Paris, 301, 11 (1985) 559-560. 
[13] N. Varopoulos. Small time Gaussian estimates of heat diffusion kernels. Part I: The semigroup technique. Bull. Sc. math. 113, 253-277 (1989).

[14] N. Varopoulos, L. Saloff-Coste \& T. Coulhon, Analysis and geometry on groups. Cambridge Tracts in Mathematics, 100. Cambridge University Press, Cambridge, 1992. xii+156 pp. ISBN: 0-521-35382-3 